\title{
Peering through the stellar wind of IGR J19140+0951 using RXTE and INTEGRAL observations
}

\section{Prat and J. Rodriguez}

DSM/IRFU/Service d'Astrophysique, CEA/Saclay, F-91191 Gif-sur-Yvette, France

E-mail: lionel.pratecea.fr

\section{Hannikainen, D.C.}

Metsähovi Radio Observatory, Helsinki University of Technology TKK, Metsähovintie 114, FI-02540 Kylmälä, Finland

\begin{abstract}
IGR J19140+0951 was discovered by the satellite INTEGRAL in 2003. This is a High Mass X-ray binary, in which the compact object is deeply embedded in the wind coming from the supergiant companion star. Due to the high level of photoelectric absorption, this type of source couldn't be detected without hard X-rays observations. The spectra obtained in the $3-80 \mathrm{keV}$ range using RXTE and INTEGRAL have allowed us to perform a precise spectral analysis of the system along its binary orbit. We were able to confirm the supergiant nature of the companion star and the neutron star nature of the compact object. Using a simple stellar wind model to describe the evolution of the photoelectric absorption, we were able to restrict the orbital inclination angle to the range $37-75^{\circ}$. Finally, we have detected a "soft excess" in at least four observations, for the first time for this source. Such soft excesses have been reported in several HMXBs in the past. We discuss the possible origin of this excess, and suggest, based on its spectral properties and occurrences close to the superior conjunction, that it may be explained as the reprocessing of the $\mathrm{X}$-ray emission originating from the neutron star by the surrounding ionized gas.
\end{abstract}

7th INTEGRAL Workshop

September 8-112008

Copenhagen, Denmark 


\section{Introduction}

High Mass X-ray Binaries (HMXBs) are binary systems consisting of a compact object orbiting a massive companion star. Prior to the launch of the INTErnational Gamma-ray Astrophysics Laboratory (INTEGRAL) in 2002, most of the known HMXBs contained a Be companion. In Betype HMXBs, the compact object emits strong X-ray flashes when it crosses the equatorial plane of the companion star, where a thick disk of matter originating from the stellar wind is present. Oand B-type stars have more isotropic stellar winds which absorb the X-ray emission of the compact object, rendering them almost undetectable below a few $\mathrm{keV}$. Thanks to its sensitivity in the soft gamma-ray range, however, INTEGRAL has found many such systems in the past few years (see e.g. $[1,2])$. In this perspective, the use of X-ray spectroscopy at different orbital phases makes it possible to probe the stellar wind, providing two-dimensional information on the density and ionization structure of the wind. For instance, the soft excess that is present in the soft X-ray spectra of many HMXBs, whose origin is still quite mysterious, is linked to the physics of the wind close to the compact object, especially the region where the fast moving stellar wind collides with the slow moving and highly ionized gas surrounding the compact object [3].

IGR J19140+0951 was discovered by INTEGRAL in March 2003 [4]. Prior to our study, it was identified as a HMXB with an orbital period of 13.552 days [5], likely composed of a neutron star [6] orbiting a B0.5 I type supergiant companion star [7]. Thus, it is a good candidate to study the properties of the obscured X-ray binaries.

\section{Spectral analysis}

Our data cover the period March 2004-September 2007 with 32 observations taken simultanously by the two satellites. The INTEGRAL data were reduced using the standard off-line Scientific Analysis (OSA) package v.7.0, and the RXTE data using the LHEASOFT package v.6.4 (for more details on the analysing process and on the results presented here, we refer to [8]). Since IGR J19140+0951 is a fairly faint source, we also corrected the spectra for the Galactic X-ray background (GXB), using the parameters measured by [9]. This correction aimed mostly at minimizing the errors on the measure of the photoelectric absorption, by removing the Galactic contribution and keeping only the absorption intrinsic to the source.

We used the ephemeris of [10] to fold the spectra, starting at phase 0 when the flux is at a minimum. The analysis of the absorption underwent by the X-ray flux along the orbit shows that phase 0 also corresponds to a minimum of the absorption (see section 4.). Thus, phase 0 corresponds to when the compact object is located between the Earth and the companion star (hereafter inferior conjunction): in this case, the X-ray flux coming from the compact object travels a shorter distance in the companion stellar wind. Phase 0.5 is therefore when the compact object is behind the companion star (superior conjunction). The fact that a minimum flux corresponds to a minimum absorption may be puzzling, but actually these two effects act on two different energy ranges: indeed, the modulation of the ASM flux is seen only in the 5-12 keV range [5], where the photoelectric absorption has only little influence. Qualitatively, when the neutron star is behind the companion star, we see directly the shock between the stellar wind and the ionized gaz surrounding the compact object, where the high-energy emission is produced. At inferior conjunction, however, 

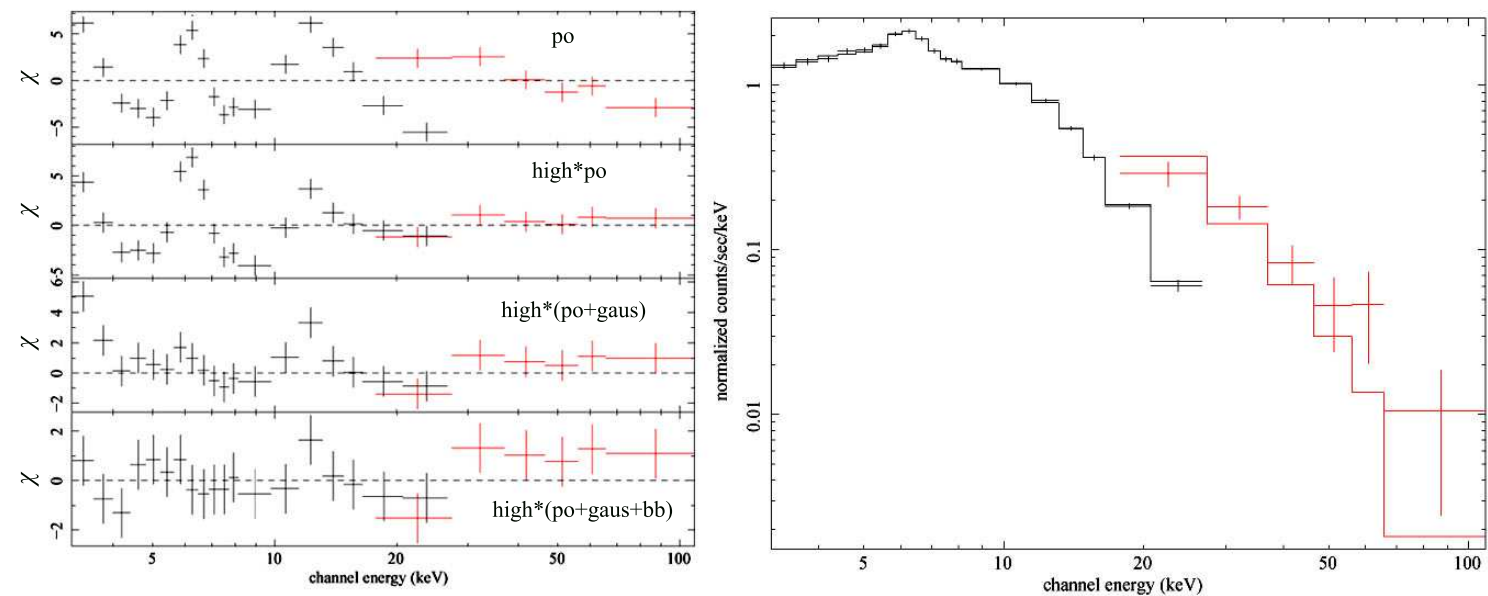

Figure 1: Left: Residuals in terms of $\sigma$ between the models used to fit the INTEGRAL+RXTE spectra. In each case the model mentioned in the panel is convolved with interstellar absorption. Po stands for powerlaw, high stands for high-energy cut-off, gaus stands for Gaussian and bb stands for black body. Right: Joint INTEGRAL+RXTE spectrum from ObsID P91083-03-02-00, corresponding to the residuals on the left. RXTE/PCA data are from 3-25 keV and INTEGRAL/ISGRI data from 20-100 keV. The best model is superposed on the spectrum.

the high energy emitting region is partially occulted by the neutron star: thus, this moment appears as a minimum flux in the ASM.

We tested several models in analysing the spectra, starting with phenomenological models. The data were well fitted using an absorbed power law combined with a high energy cut-off. An iron fluorescent line at $6.4 \mathrm{keV}$ is present in the observations where the source is detected at a high enough significance. Note that the high energy cut-off is only detected in the observations where the source flux is sufficiently high, thus giving enough precision at the high energy end of the spectra. The presence of this cut-off allowed us to identify the emission process as thermal Comptonization, which is quite common for this type of source. This is also confirmed by the good fitting results obtained with the more physical Comptonization model COMPTT [11], in XSPEC notations. A joint RXTE-INTEGRAL spectrum is shown in Fig. 1, right.

The photoelectric absorption $N_{\mathrm{H}}$ undergoes strong variations, well correlated with the orbital phase (Fig. 2, left). Since the orbit of the X-ray source is probably almost circular, one possible explanation for the observed change in $N_{\mathrm{H}}$ relies on an inclined orbit for the system. Qualitatively, when the neutron star is behind its companion its light has to travel a longer distance through a denser part of the stellar wind, so the absorption in the soft X-rays increases. Therefore, at the superior conjunction we should observe a maximum in the absorption, which is indeed the case.

The unabsorbed flux undergoes strong variations that are not correlated with the orbital phase; this may be a consequence of the details of the accretion process. The stellar wind is not perfectly homogeneous and the accretion onto the neutron star, following the magnetic field line to the magnetic poles, is a highly unstable and complicated process (see e.g. [12] and references therein, for a review of the emission processes). Since our observations are spread over several years, it is not surprising that the source flux is highly variable. This may also be a direct consequence of 

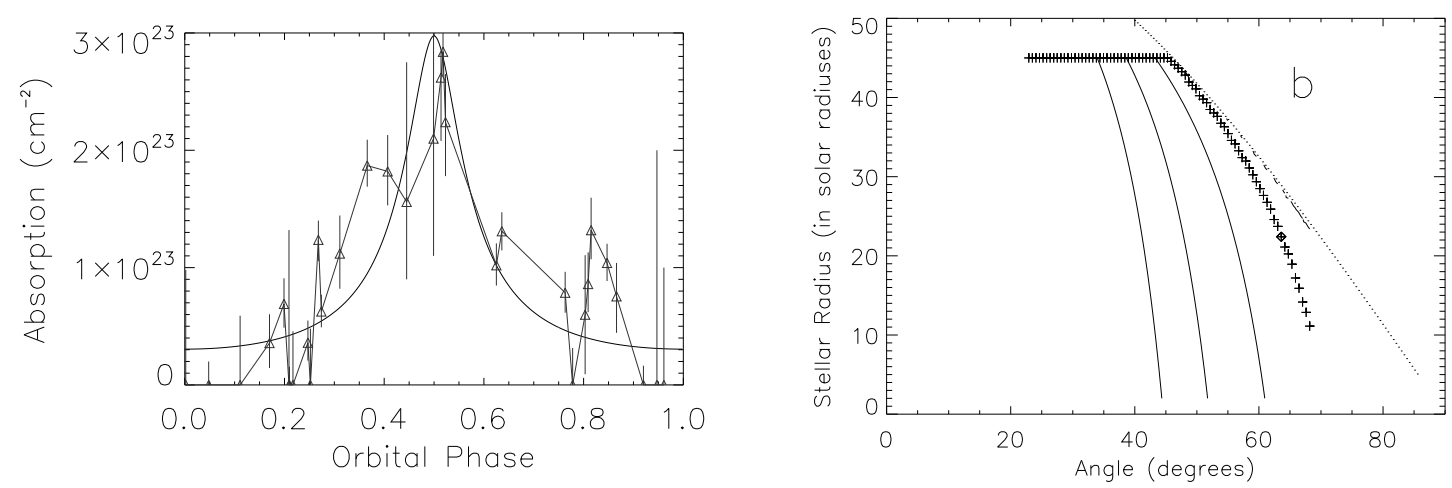

Figure 2: Left : Model absorption (continuous line) and experimental absorption ( $\triangle$ symbols), for the stellar model with parameters $\mathrm{M}_{\star}=20 \mathrm{M}_{\odot}, \mathrm{R}_{\star}=21 \mathrm{R}_{\odot}$ and $\beta=0.5$. Phase 0 corresponds to when the compact object is located between the Earth and the companion star (inferior conjunction). Right : Most probable orbital inclination, as a function of the companion radius, for a typical set of parameters. The + symbols correspond to the most probable orbital inclination, confidence contours are drawn at the 25, 68 and 90\% confidence levels. The dotted line is the "eclipse limit": points above this line are excluded as it would imply an eclipse of the neutron star by its companion, which is not observed.

the clumpiness of the stellar wind: depending on the times of the observation, the compact object may be in the course of accreting a denser portion of the wind [13]. Unfortunately, the rather short exposure times and the too long periods between successive observations restrained precise characterisations of this clumpiness.

\section{Soft Excess detection}

But a more interesting characteristic is the detection of a so called "soft excess". During our study, some spectra exhibited an excess in the soft X-ray part of the spectra, which we modeled by adding a black body component to the model. This feature has already been observed in many $\mathrm{X}$-ray binaries, and several models have already been proposed to explain it (see [3] for a review). In the case of IGR J19140+0951, it can probably be linked to the immediate environment of the neutron star, and explained qualitatively by the following scenario. The stellar wind of massive stars is mostly accelerated by bound-bound transitions of atoms. The neutron star, due to its high energy emission, ionizes the surrounding material, so the already ionized gas around the neutron star is no longer accelerated by the stellar radiation field. When the compact object moves along its orbit, the hot gas will gradually be overtaken by the stellar wind. This will lead to the formation of a "tail" trailing the neutron star.

As the gas in this tail is ionized, its absorption will be lower, but its precise effect on the emission is not clear. Since the soft excess is only visible around the superior conjunction (between phases 0.40 and 0.75 ), one possibility is that the hard X-ray emission may be scattered around the tail. The precise study of this effect, however, would require better spectral resolution in the soft $\mathrm{X}$-ray range than what is available on RXTE. 


\section{Wind model}

In order to constrain several parameters for the system, we used a simple " $\beta$-law" wind model: it adequately describes the strong stellar winds of B-type stars, which is usually taken to be stationary and spherically symmetric. See e.g. [14] for a precise description of the method. Using the evolution of the photoelectric absorption, we were able to constrain the inclination of the system and the mass-loss rate of the companion star. For the most probable stellar radius of $21 R_{\odot}$, the lower inclination limit is constrained between 37 and $42^{\circ}$ (Fig. 2, right), with $\chi^{2}$ being at a minimum in the range $55-63^{\circ}$. Fig. 2, left, shows the best-fit model against the experimental normalized data, with a good agreement. The mass-loss rate, $\dot{M}_{\star}$, is constrained to the range $0.6-1.0 \times 10^{-7}$ $\mathrm{M}_{\odot} /$ year, which is consistent with what is expected for a B0.5 type supergiant.

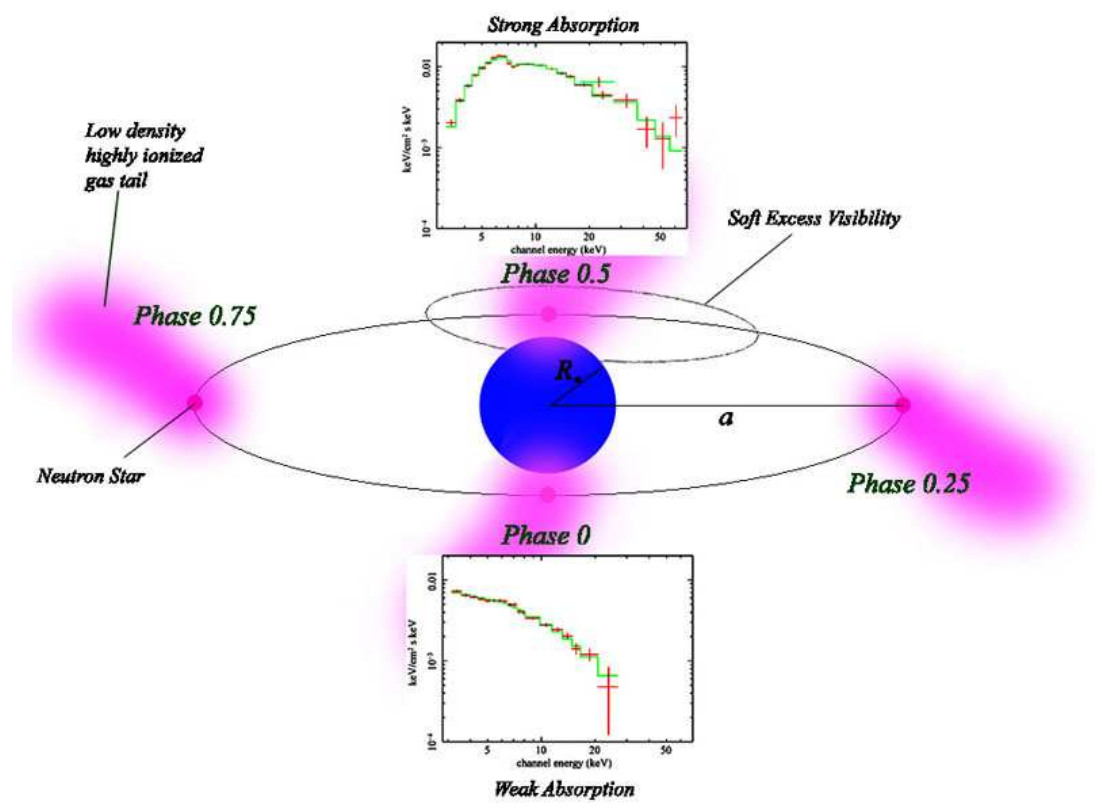

Figure 3: Diagram of IGR J19140+0951 as it could be seen from the Earth. The orbital inclination of the system is taken to be $\sim 65^{\circ}$.

Fig. 3 summarizes schematically our main results. We found that photoelectric absorption highly correlates with the orbital phase of the system. Using a simple stellar wind model we found a rather high orbital inclination, $\sim 65^{\circ}$. We have detected a soft excess in some observations, just before the superior conjunction, in the area indicated on the figure. This may be explained by a cloud of highly ionised gas surrounding the neutron star. Because of its ionisation, this gas is less accelerated by the stellar wind and, while being gradually overtaken by the wind, tends to form a "tail". This tail could scatter the hard X-ray emission from the compact object and thus explain the soft excess feature. However, these results remain qualitative, as a precise determination of the geometry of the stellar wind would require a better sensitivity in the soft X-ray range, which could be achieved for instance with the use of the XMM or Chandra satellites. 


\section{Conclusions}

The study of X-ray binaries is challenging since it is often difficult, if not impossible, to identify their visible and infrared counterparts. Even if an infrared counterpart were observed, the distance to some systems prohibits the measurement of the orbital characteristics. Our study shows that X-ray observations can overcome these limitations and produce very precise inferences. The RXTE and INTEGRAL observations of IGR J19140+0951 have led to good measurements of the orbital period of the system, constraints on its inclination angle, and the diagnostic of the type of the companion star (supergiant $\mathrm{O}$ or $\mathrm{B}$ )

Moreover, we can use the compact object to probe the stellar wind of the companion. In the case of IGR J19140+0951, we diagnosed the wind density, the probable presence of clumps, and the wind structure around the neutron star. More precise observations could lead to constraints on the mass and radius of the companion, and better constraints on the stellar wind. This allows the study of new INTEGRAL sources, either distant or highly absorbed, and ultimately the determination of new useful data for X-ray binary evolution scenarios.

\section{References}

[1] Liu, Q. Z., van Paradijs, J., van den Heuvel, E. P. J., 2006, A\&A, 455, 1165

[2] Bodaghee A., Courvoisier T. J.-L., Rodriguez J., et al., 2007, A\&A, 457, 585

[3] Hickox R. C., Narayan R., \& Kallman T. R., 2004, ApJ, 614, 881

[4] Hannikainen D., Rodriguez J., \& Pottschmidt K., 2003, IAUC, 8088

[5] Wen L., Levine A., Corbet R., \& Bradt H., 2006, ApJS, 163, 372

[6] Rodriguez J., Cabanac C., Hannikainen D., et al., 2005, A\&A, 432, L17

[7] Hannikainen D., Rawlings M. G., Muhli P., et al., 2007, MNRAS, 380, 665

[8] Prat L., Rodriguez J., \& Hannikainen D., 2008, MNRAS, 389, 301

[9] Valinia, A., \& Marshall, F. E., 1998, ApJ, 505, 134

[10] Corbet R., Hannikainen D., Remillard R., 2004, Atel, 269

[11] Titarchuk L., 1994, ApJ, 434, 570

[12] Takata J., Shibata S., Hirotani K., \& Chang H.-K., 2006, MNRAS, 366, 1310

[13] Blay P., Martínez-Núñez S., Negueruela I., Pottschmidt K., Smith D. M., et al., 2008, [astro-ph/08064097]

[14] Levine A. M., Rappaport S., Remillard R., \& Savcheva A., 2004, ApJ, 617, 134 